ARTICLE OPEN

\title{
Single cell analysis reveals inhibition of angiogenesis attenuates the progression of heterotopic ossification in $M k x^{-1-}$ mice
}

Junxin Lin ${ }^{1,2,3}$, Yuwei Yang ${ }^{1,3}$, Wenyan Zhou ${ }^{1,3}$, Chao Dai ${ }^{3}$, Xiao Chen (iD ${ }^{1,2,3,4}$, Yuanhao Xie ${ }^{1,2}$, Shan Han ${ }^{1,3}$, Huanhuan Liu $^{1,3}$, Yejun Hu $^{1,2}$, Chenqi Tang ${ }^{1,2}$, Varitsara Bunpetch ${ }^{1,3}$, Dandan Zhang ${ }^{5}$, Yishan Chen ${ }^{1,3}$, Xiaohui Zou ${ }^{1,3,6}$, Di Chen ${ }^{3,7}$, Wanlu Liu ${ }^{1,3 凶}$ and Hongwei Ouyang $\mathbb{D}^{1,2,3,4 凶}$

Tendon heterotopic ossification $(\mathrm{HO})$ is characterized by bone formation inside tendon tissue, which severely debilitates people in their daily life. Current therapies fail to promote functional tissue repair largely due to our limited understanding of HO pathogenesis. Here, we investigate the pathological mechanism and propose a potential treatment method for HO. Immunofluorescence assays showed that the Mohawk (MKX) expression level was decreased in human tendon HO tissue, coinciding with spontaneous $\mathrm{HO}$ and the upregulated expression of osteochondrogenic and angiogenic genes in the tendons of $M k^{-1-}$ mice. Single-cell RNA sequencing analyses of wild-type and $M k^{-1-}$ tendons identified three cell types and revealed the excessive activation of osteochondrogenic genes during the tenogenesis of $M k^{-1-}$ tendon cells. Single-cell analysis revealed that the gene expression program of angiogenesis, which is strongly associated with bone formation, was activated in all cell types during HO. Moreover, inhibition of angiogenesis by the small-molecule inhibitor BIBF1120 attenuated bone formation and angiogenesis in the Achilles tendons of both Mkx mutant mice and a rat traumatic model of HO. These findings provide new insights into the cellular mechanisms of tendon $\mathrm{HO}$ and highlight the inhibition of angiogenesis with BIBF1120 as a potential treatment strategy for HO.

Bone Research (2022)10:4 ～； https://doi.org/10.1038/s41413-021-00175-9

\section{INTRODUCTION}

The tendon anchors muscle to bone and transmits the forces generated by musculoskeletal tissues to enable body movement. Increasing incident rates of tendon injuries due to strenuous exercise or age-related degeneration are becoming increasingly challenging for medical personnel. Tendon heterotopic ossification ( $\mathrm{HO}$ ), which is characterized by endochondral bone formation in tendons, is the major histological feature of tendon injury or tendinopathy at later stages ${ }^{1,2}$. However, the lack of knowledge about the pathogenesis of $\mathrm{HO}$ has hampered the development of effective therapies. Current clinical treatment options for $\mathrm{HO}$ are limited to physical therapy, the use of nonsteroidal anti-inflammatory drugs and surgical excision of the ectopically formed bone, which may cause frequent complications and a high recurrence rate ${ }^{3}$. Therefore, better understanding the cellular and molecular mechanisms of $\mathrm{HO}$ will provide new clues for novel therapeutic or prophylactic approaches.

Progenitor cells contributing to tendon $\mathrm{HO}$ have been evaluated by lineage tracing ${ }^{4}$. Recent studies have shown that Scleraxis (Scx)-expressing tendon progenitor cells contribute to $\mathrm{HO}$ in trauma or hyperactive bone morphogenetic protein (BMP) induced heterotopic ossification models. Constitutive expression of the active form of the type I BMP receptor ACVR1 in Scx-expressing cells results in a clear $\mathrm{HO}$ phenotype in mouse joints and tendons, even in the absence of injury. These pioneering studies indicate that the ectopic activation of osteogenic signaling confers osteogenic differentiation potential in tendon progenitor cells. However, various subpopulations of tendon resident cells exist ${ }^{5-8}$, and their contributions to the $\mathrm{HO}$ process has not been studied.

Mohawk (Mkx) is a tendon-specific transcription factor, and its mutation causes tendon $\mathrm{HO}$ in mice and rats ${ }^{9-13}$. Using $M k x$ knockout rodent models, recent studies revealed that tendonderived cells exhibited increased expression levels of chondrogenic markers and accelerated chondrogenic and osteogenic differentiation during the progression of $\mathrm{HO}^{13,14}$. These studies provide critical information about the tendon $\mathrm{HO}$ phenotype and suggest that $M k x$ knockout animals can be employed as an appropriate model to study the cellular and molecular mechanisms governing the progression of $\mathrm{HO}$.

In this study, we utilized $M \mathrm{kx}^{-/-}$mice as a model of tendon $\mathrm{HO}$ to explore its pathogenesis and potential treatments. Our results at the single-cell level suggest that tendon progenitor cells commit to an osteoblastic fate during $\mathrm{HO}$ progression. Moreover, tendon $\mathrm{HO}$ was shown to be associated with the

\footnotetext{
${ }^{1}$ Dr. Li Dak Sum \& Yip Yio Chin Center for Stem Cells and Regenerative Medicine, and Department of Orthopedic Surgery of the Second Affiliated Hospital, Zhejiang University School of Medicine, Hangzhou, China; ${ }^{2}$ Department of Sports Medicine, Zhejiang University School of Medicine, Hangzhou, China; ${ }^{3}$ Zhejiang University-University of Edinburgh Institute, Zhejiang University School of Medicine, and Key Laboratory of Tissue Engineering and Regenerative Medicine of Zhejiang Province, Hangzhou, China; ${ }^{4}$ China Orthopedic Regenerative Medicine Group (CORMed), Hangzhou, China; ${ }^{5}$ Department of Pathology, Zhejiang University School of Medicine, Hangzhou, China; ${ }^{6}$ Clinical Research

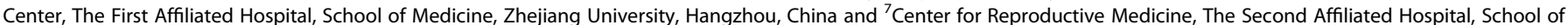
Medicine, Zhejiang University, Hangzhou, China

Correspondence: Wanlu Liu (wanluliu@intl.zju.edu.cn) or Hongwei Ouyang (hwoy@zju.edu.cn)
}

Received: 27 August 2020 Accepted: 1 September 2021

Published online: 07 January 2022 


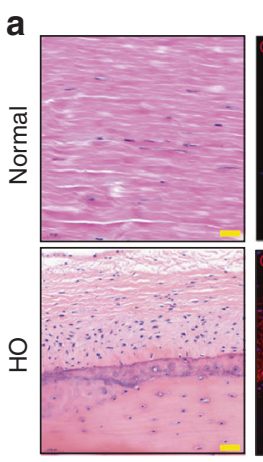

b

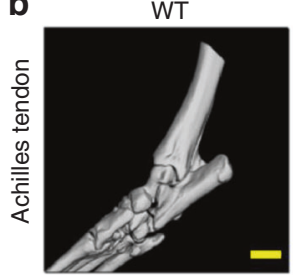

d

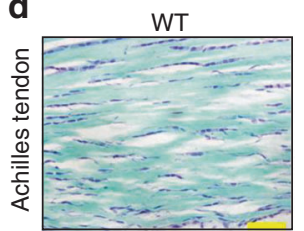

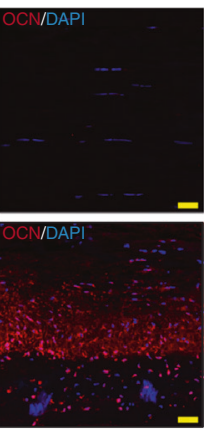

$M k x^{-1-}$
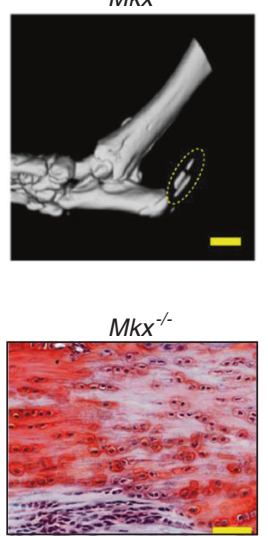

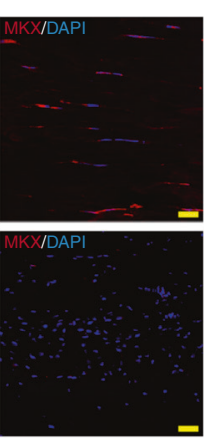

C
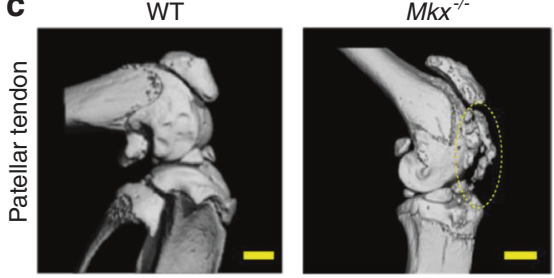

e
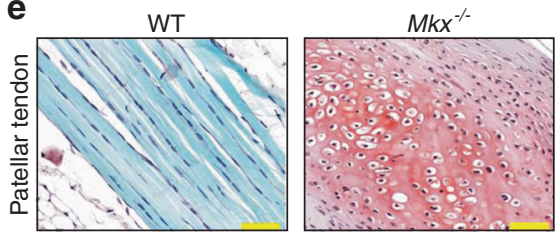

Fig. 1 Mkx deficiency causes tendon heterotopic ossification. a HE (left) and immunofluorescence staining of OCN (middle) and MKX (right) in the tendons of normal individuals and $\mathrm{HO}$ patients. Scale bar, $50 \mu \mathrm{m}$. b, c Micro-CT examination of Achilles tendons (b) and patellar tendons (c) from WT and $\mathrm{Mkx}^{-1-}$ mice. Scale bar, $1 \mathrm{~mm}$. d, e Safranin O-Fast green staining of Achilles tendons (d) and patellar tendons (e) from WT and $M k x^{-1-}$ mice. Scale bar, $50 \mu \mathrm{m}$

upregulated expression of proangiogenic genes in all tendon cell clusters. Inhibition of angiogenesis by the small molecule BIBF1120 attenuated bone formation and angiogenesis in both the $M \mathrm{kx}^{-/-}$mouse degenerative $\mathrm{HO}$ model and the rat traumatic model of $\mathrm{HO}$.

\section{RESULTS}

MKX is suppressed in human $\mathrm{HO}$, and Mkx knockout leads to $\mathrm{HO}$ in mouse tendons

To assess the potential involvement of MKX in human $\mathrm{HO}$ pathogenesis, we evaluated the expression of MKX in the tendons of normal individuals and $\mathrm{HO}$ patient by immunofluorescence analysis. Hematoxylin and eosin (H\&E) staining revealed $\mathrm{HO}$ in human tendons with clear tendon layers, well-developed bone and their interface. Osteocalcin-positive $\left(\mathrm{OCN}^{+}\right)$osteoblasts were present at the interface and bone region. The MKX protein was detected in tendon cells from normal individuals (Fig. 1a). However, in the HO samples, the expression of MKX was dramatically decreased (Fig. 1a), indicating that MKX is involved in tendon $\mathrm{HO}$ in humans.

Previous studies reported $\mathrm{HO}$ in the Achilles tendons of $M k x-$ deficient rats and mice ${ }^{13,14}$, but whether tendons from other sites are affected and how $\mathrm{HO}$ develops remain unknown. To further characterize the tendon heterotopic ossification phenotype, we analyzed wild-type (WT) and $\mathrm{Mkx}^{-1-}$ mice by microcomputed tomography (micro-CT) and histological staining. As expected, ectopic bone was clearly observed in the Achilles tendons of $\mathrm{MkX}^{-1-}$ mice, confirming previously reported $\mathrm{HO}$ phenotype (dotted circles in Fig. 1b) ${ }^{14}$. Importantly, the $\mathrm{Mkx}^{-/-}$mice also exhibited a clear and detectable heterotopic bone formation phenotype in their patellar and tail tendons at 8 weeks of age (dotted circles in Fig. 1c and Supplementary Fig. S1). To further confirm the $\mathrm{HO}$ phenotype in $\mathrm{Mkx}^{-1-}$ tendons, we performed safranin $\mathrm{O}$ and fast green (SOFG) staining. In WT Achilles and patellar tendons, clear elongated nuclei labeled by dark blue staining lined the extracellular matrix (stained a light blue), which is a characteristic of tendon architecture (Fig. 1d, e). In contrast, abundant proteoglycans, indicated by red staining, and chondrocytes with rounded nuclei were observed in the Achilles and patellar tendons of $M k x$ mutants, confirming the characteristic chondral lesions and endochondral ossification observed in $\mathrm{HO}$ (Fig. 1d, e). These observations confirm that $M k x$ deficiency leads to $\mathrm{HO}$ in both Achilles, patellar and tail tendons. Taken together, these results indicate that $M k x$ knockout mice represent a suitable model for elucidating the pathogenic mechanisms of tendon $\mathrm{HO}$.

Upregulation of osteochondrogenic and angiogenic genes in $\mathrm{HO}$ tendons

To investigate the molecular mechanism of tendon $\mathrm{HO}$, we first performed bulk RNA sequencing (RNA-seq) on tail tendons isolated from 4-week-old $M k^{-1-}$ and WT mice. We then analyzed differentially expressed genes (DEGs) between $M k^{-/-} \mathrm{HO}$ and WT control tissues to identify genes that are potentially involved in $\mathrm{HO}$ (using cutoff thresholds of a fold change greater than 2 and an FDR less than 0.05). DEG analysis revealed 393 upregulated genes and 283 downregulated genes in Mkx-deficient tendons (Fig. 2a). Gene ontology (GO) analysis suggested that the genes with upregulated expression in $\mathrm{Mkx}^{-/-}$tendons were associated with cell adhesion, muscle contraction, angiogenesis, bone regeneration, and ossification (Fig. 2b), while genes with downregulated expression in $\mathrm{Mkx}^{-/-}$tendons were enriched in collagen fibril organization, cell adhesion, extracellular matrix organization, response to mechanical stimuli, and tendon formation (Fig. 2c). 


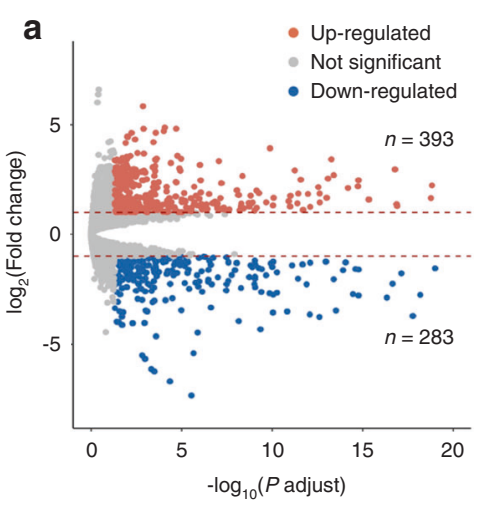

b

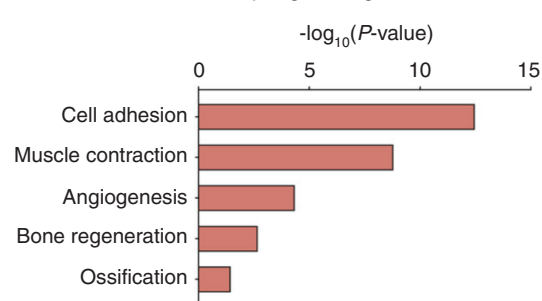

d
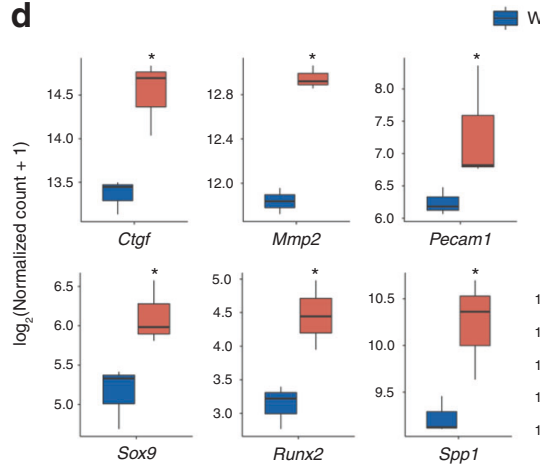

WT $M k x$
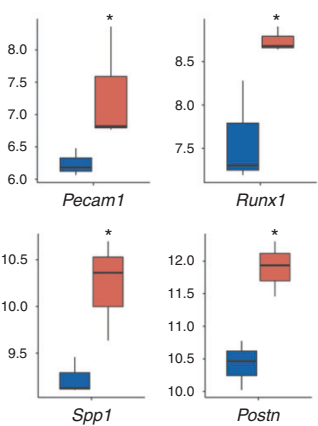

C

GO terms of down-regulated genes in $M k x^{-/}$

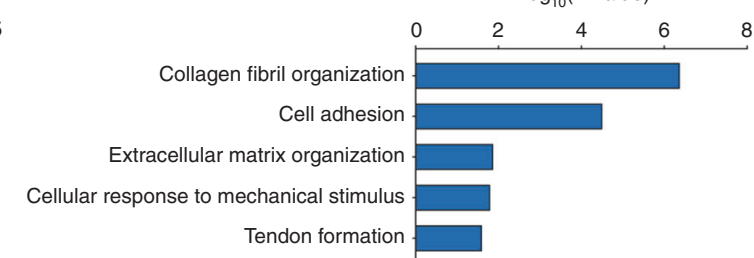

Fig. 2 Transcriptomic analysis of WT and $M k^{-1-}$ tendons. a Volcano plot of differentially expressed genes between WT and $M k x^{-1-}$ tendons. b Representative enriched GO terms corresponding to upregulated genes in $M k x^{-/-}$tendons. c Representative enriched GO terms corresponding to downregulated genes in $\mathrm{Mkx}^{-1-}$ tendons. d Boxplots of the expression levels of genes related to angiogenesis (Ctgf, $\mathrm{Mmp2}$, Pecam 1 and Runx1) and osteochondrogenesis (Sox9, Runx2, Spp1 and Postn) in WT and Mkx ${ }^{-1-}$ tendons. *:p $<0.05$

To identify potential signaling pathways regulated by Mkx, we performed Kyoto Encyclopedia of Genes and Genomes (KEGG) analysis of the genes that were upregulated upon Mkx knockout. The top 20 enriched pathways are shown in Supplementary Fig. S2a. Many genes were highly and specifically clustered in the Wnt, PI3K-Akt and Rap1 signaling pathways, which are importantly associated with the physiological and pathological features of angiogenesis ${ }^{15}$. Further examination of factors known to be associated with angiogenesis (Fig. 2d, above and Supplementary Fig. S2b) and osteochondrogenesis (Fig. 2d, below) revealed that these genes exhibited significantly upregulated expression in $\mathrm{Mkx}^{-1-}$ tendons (Fig. 2d). Taken together, these findings indicate that genes related to angiogenesis and osteochondrogenesis are upregulated in $\mathrm{Mkx}^{-1-}$ tendons.

Cellular profiles and transcriptomic signatures of WT and $\mathrm{MkX}^{-1-}$ tendons at the single-cell level

Given that tendon tissues are composed of different cell populations and that bulk RNA-seq revealed only the average expression levels in all cells, we next examined the expression profiles at the single-cell level to determine the cell populations contributing to the progression of $\mathrm{HO}$. To do this, we isolated tail tendons from 4-week-old WT and $\mathrm{Mkx}^{-/-}$mice and performed single-cell RNA-seq (scRNA-seq) using the Fluidigm C1 system with high-throughput integrated fluidic circuits (HT IFCs). In total, we captured 486 single cells, of which 466 cells passed our quality control criteria (materials and methods) and were included in further analyses. To compare WT and $M k x^{-/-}$cells directly, we utilized a recently developed computational method (Seurat alignment workflow; SAW) for the integrated analysis of scRNAseq data obtained under different conditions ${ }^{16}$. Unsupervised graph-based clustering was performed on the integrated dataset of both WT and $\mathrm{Mkx}^{-1-}$ cells, and the result was visualized using ( $t$-distributed stochastic neighbor embedding (t-SNE)). Three distinct cell clusters were identified in both WT and $\mathrm{Mkx}^{-1-}$ tendons (Fig. 3a). More importantly, WT and $\mathrm{Mkx}^{-/-}$cells were intermingled with each other in all three populations (Fig. 3b), suggesting that no population shift occurred in $\mathrm{HO}$ tendons at this stage. Consistently, the cell population compositions were comparable between WT and $M k x^{-1-}$ tendons (Fig. 3c). Taken together, the above analyses ruled out the possibility of cell population shifting as the main mechanism underlying $\mathrm{HO}$ progression.

To annotate the cells, marker genes representing each cluster were identified using Seruat ${ }^{16}$. For each cell cluster, we identified 126, 25, and 76 marker genes (Fig. 3d). Cells highly expressing markers of mesenchymal progenitor cells, such as Cd44, Thy 1, and Ly6a, were annotated as tendon progenitor cells (TPCs) (Fig. 3d). TPCs also expressed high levels of Fst/1 (Supplementary Fig. S3a), which has been reported to be upregulated at the onset of tenogenic mesenchymal stem cell differentiation ${ }^{17}$. Cells with high levels of Itm2a, a type II integral membrane protein involved in mesenchymal stem cell differentiation ${ }^{18-20}$, were annotated as tenoblasts (TBs) (Fig. 3d and Supplementary Fig. S3b). Cells enriched for extracellular matrix genes secreted by mature tendon cells, such as Col11a2, Col1a1, Col1a2, Sparc, Tnmd and Fmod (Fig. 3d and Supplementary Fig. S3C), were designated as tenocytes (TCs) ${ }^{21-23}$. We also validated the expression of one representative marker for each cell type (Fstl1, Imt2a, Col1) by immunofluorescence, confirming the existence of these cell types in both WT and $M k^{-/-}$ tendons (Supplementary Fig. S3d). We then performed GO analyses of these marker genes in each cell type (Fig. 3d). TPCs were enriched for GO terms such as cell adhesion, extracellular matrix organization and cell migration, and TBs were enriched for the GO terms cell differentiation and cell adhesion. Intriguingly, TCs were enriched for GO terms such as collagen fibril organization, cartilage development and ossification, indicating that a proportion of tendon cells might easily initiate and contribute to endochondral bone formation (Fig. 3d). Overall, we identified three distinct cell subpopulations in both WT and $M k x^{-1-}$ tendons. 
a

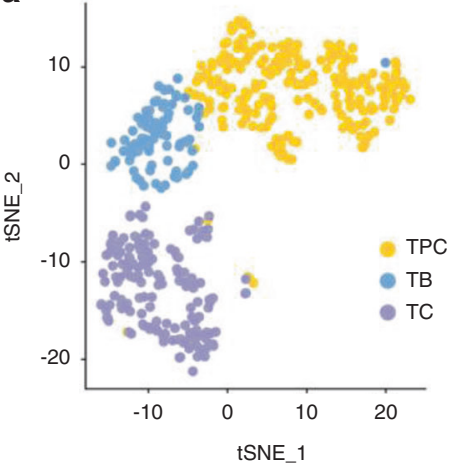

b

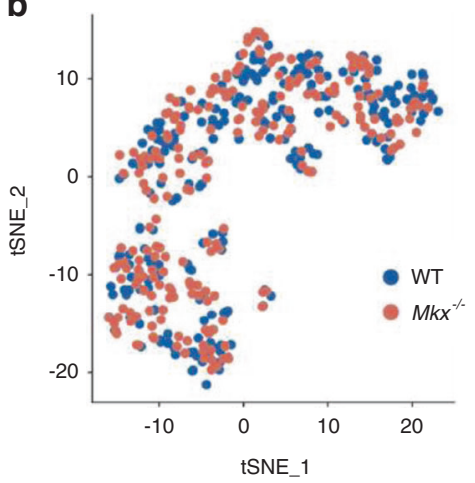

C

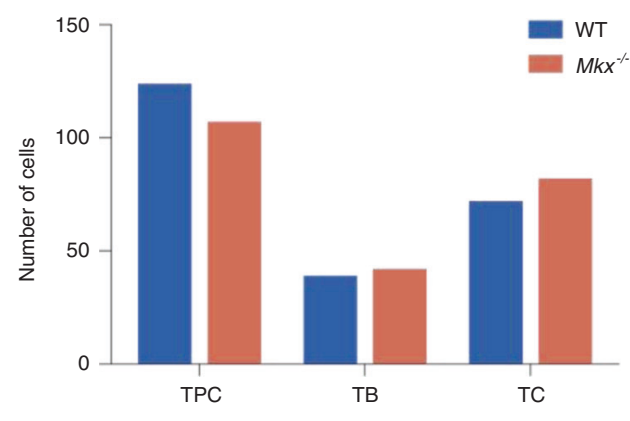

d

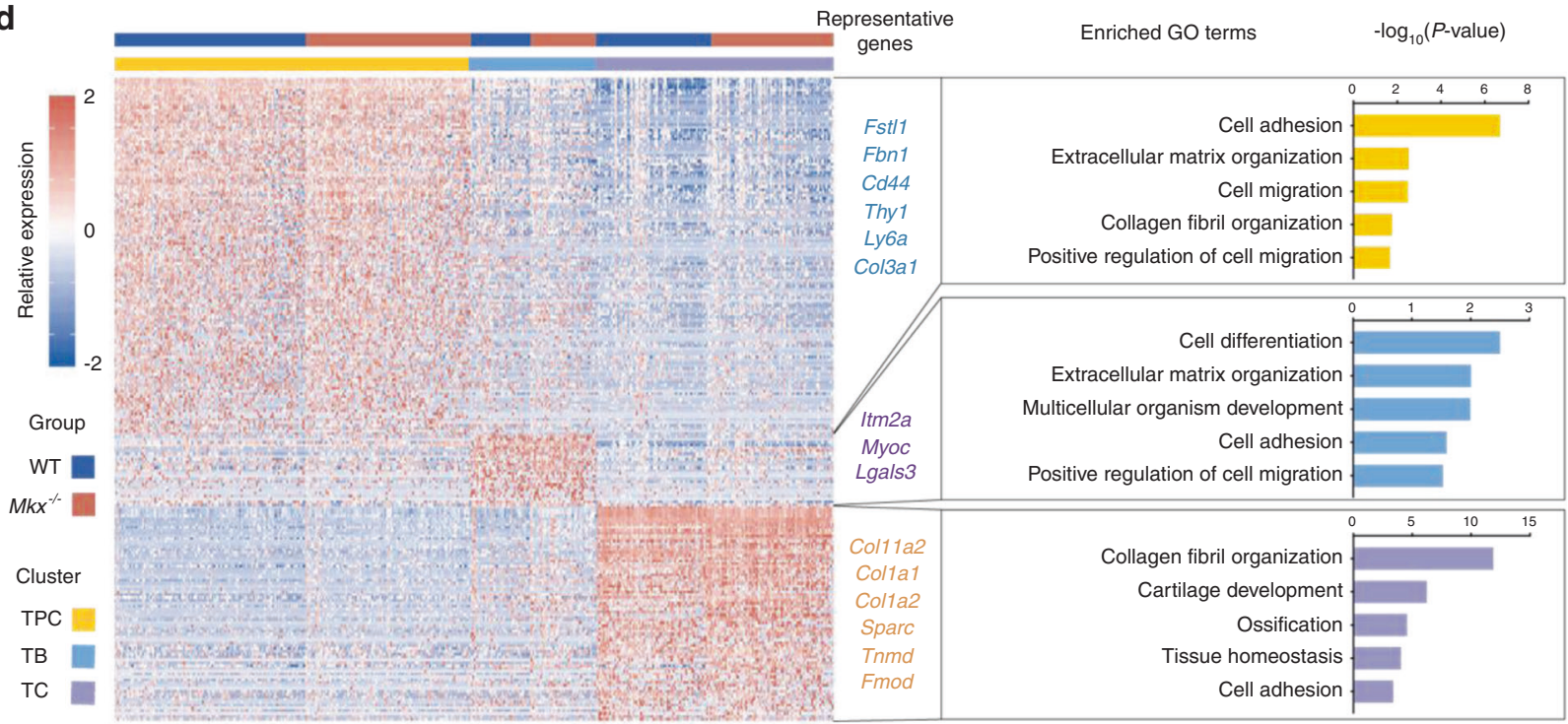

Fig. 3 Single-cell profiles of WT and $M k x^{-/-}$tendons. $\mathbf{a}, \mathbf{b}$ t-SNE plot of WT and $M k x^{-/-}$tendon cell clusters inferred from scRNA-seq data. The plots were visualized using CC1-CC4 and are colored by cell type (a) or genotype (b). CC: canonical vectors. c Numbers of WT and Mkx ${ }^{-1-}$ cells in each cell cluster. $\mathbf{d}$ Heatmap showing the marker genes for each cell cluster (left) and representative GO terms corresponding to these marker genes (right). TPCs tendon progenitor cells, TBs tenoblasts, TCs tenocytes

Trajectory analyses reveal ectopic activation of osteochondrogenic genes in $\mathrm{MkX}^{-1-}$ cells during tenogenesis

We next evaluated the gene expression dynamics during the heterotopic ossification process by analyzing the pseudotemporal trajectory using Monocle ${ }^{24}$. In both WT and $M k^{-1-}$ tendon cells, we observed an enrichment of TPCs at the start of the pseudotemporal trajectory, which passed through an intermediate state dominated by TBs and ultimately transitioned to the terminal state, mainly consisting of TCs (Fig. 4a, b). Differentially expressed genes throughout WT or $M k^{-1-}$ tendon differentiation were clustered into three gene sets, reflecting the early, middle and late differentiation stages (Fig. 4c, d). The pseudotemporal kinetics of representative early (Ly6a, Tppp3), middle (Itm2a, Lgals3) and late (Tnmd, Col1a1) stage markers showed similar expression patterns in WT and $\mathrm{Mkx}^{-/-}$cells, suggesting that the key gene expression program required for tenogenesis was, to a certain extent, maintained in $\mathrm{Mkx}^{-1-}$ cells (Fig. 4e, f).

We next examined whether the expression levels of osteochondrogenic genes were altered in $\mathrm{Mkx}^{-1-}$ tendon cells. Interestingly, osteochondrogenic marker genes (Spp1, Ogn, Mgp, and (omp) showed similar increasing expression trends from the early to the late stage of differentiation in WT and $\mathrm{Mkx}^{-1-}$ tendon cells (Fig. $4 \mathrm{~g}, \mathrm{~h}$ ). Nevertheless, the upregulation of osteogenic genes seemed to occur earlier in $\mathrm{Mkx}^{-/-}$cells than in WT cells
(Fig. 4g, h). In addition, the expression levels of these genes were relatively higher in $\mathrm{Mkx}^{-1-}$ cell clusters than in WT cells (Fig. $4 \mathrm{i}$ ). To further investigate the changes in the osteochondrogenic gene expression program in $M k^{-1-}$ cells, we performed gene set score analysis to determine whether a specific set of genes was expressed in a specific cell type at higher than expected levels. We found that the expression levels of genes associated with osteoblast development were significantly increased in $\mathrm{Mkx}^{-1-}$ TPCs, while genes linked to ossification and bone mineralization were highly expressed in both WT and $M_{k x^{-/}}$TBs and TCs (Supplementary Fig. S4a, b and Supplementary Table S1). Collectively, these analyses indicated that the heterotopic ossification of $\mathrm{Mkx}^{-1-}$ tendons was associated with the excessive activation of osteochondrogenic genes as early as the TPC stage and not with the de novo activation of these genes.

Increased angiogenic gene expression and vascularity in $\mathrm{Mkx}^{-/-}$ tendons

To further explore the effects of Mkx knockout on the gene expression levels in different cell types, we analyzed the DEGs between WT and $\mathrm{Mkx}^{-1-}$ tendons for each cell cluster (Fig. 5a-c). We performed GO term analysis of genes that were upregulated in $\mathrm{MkX}^{-/-}$cells for each cell type (Fig. 5d-f). Interestingly, angiogenesis-related genes were consistently enriched in all 


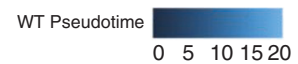

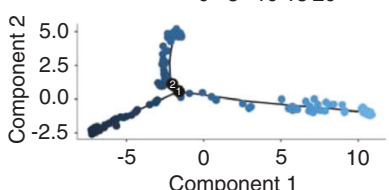

WT-TPC WT-TB WT-TC

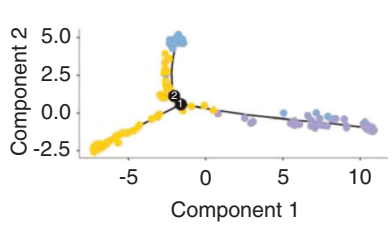

b

$\begin{array}{llll}\text { Mkx Pseudotime } & & \\ & 0 & 5 \quad 101520\end{array}$

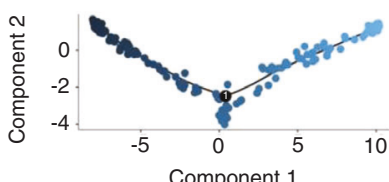

$M k x^{*}-\mathrm{TPC} \odot M k x^{*}-\mathrm{TB} \odot M k x^{\%}-\mathrm{TC}$

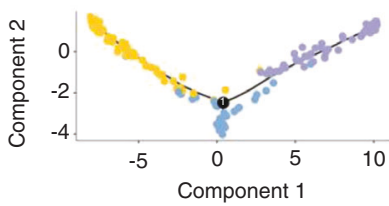

g

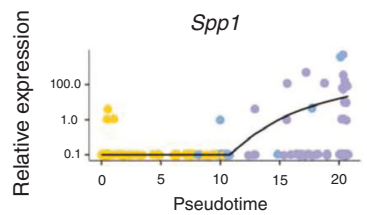

h

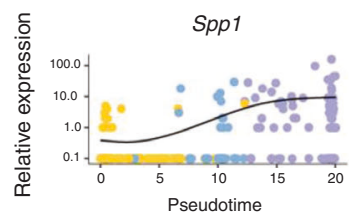

i

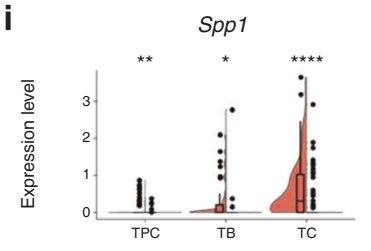

C

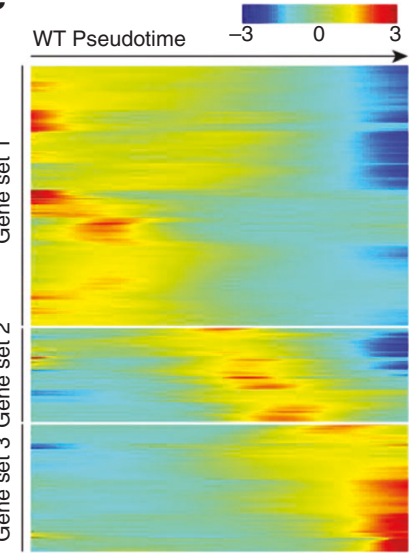

d

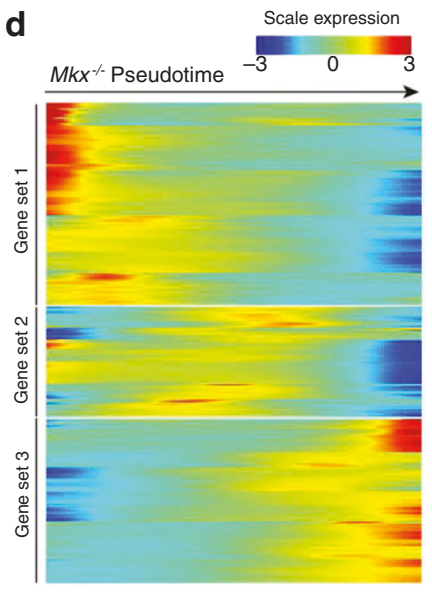

e

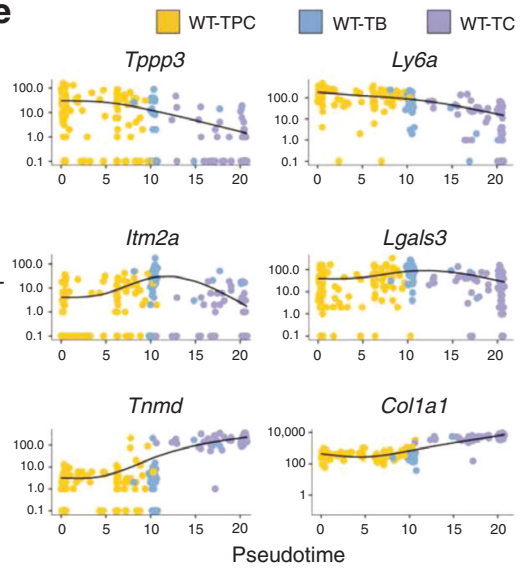

f

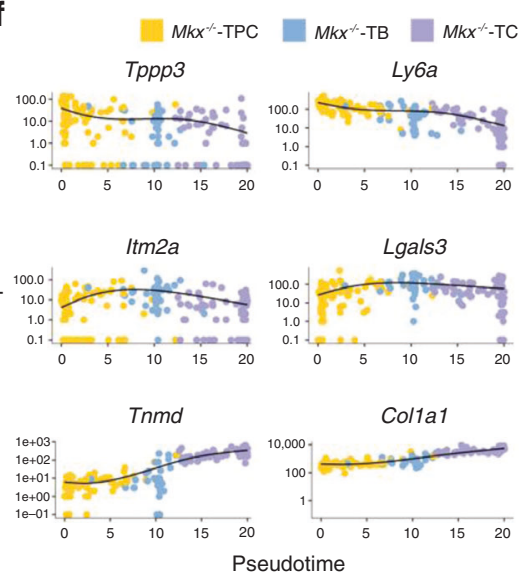

WT-TPC WT-TB WT-TC
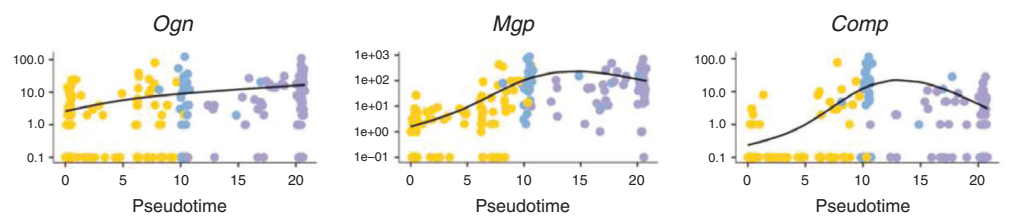

$M k x^{\prime-T P C} \square k x^{\prime}-\mathrm{TB} \square M k x^{\prime-T C}$
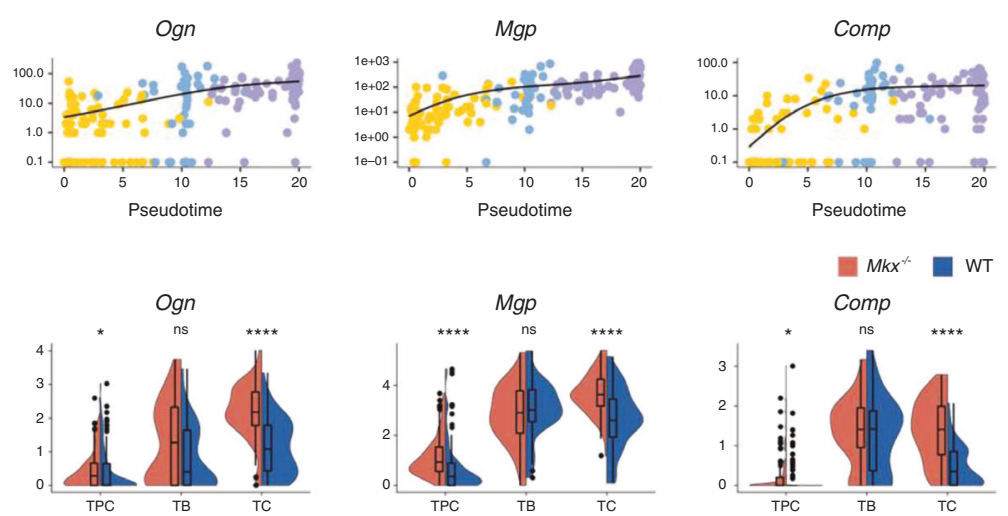

Fig. 4 Pseudotime analysis revealed shifted gene expression programs in $\mathrm{Mkx}^{-/-}$tendon cells. a, b Order of the WT (a) and $\mathrm{Mkx}^{-/-}$(b) cells along the differentiation trajectory based on pseudotime values and cell clusters. c, $\mathbf{d}$ Gene expression dynamics along the WT (c) and $M k x^{-/-}$ (d) differentiation trajectories. Genes (row) are clustered and cells (column) are ordered according to pseudotime development. e, f Kinetic diagrams showing the expression of early, middle and late tenogenesis markers during the differentiation of WT (e) and $M k x^{-1-}$ (f) cells. $\mathbf{g}$, $\mathbf{h}$ Kinetic diagrams showing the expression of osteochondrogenic markers during the differentiation of WT $(\mathbf{g})$ and $M k x^{-1-}$ (h) cells. i Violin plots showing the Spp1, Ogn, Mgp, and Comp expression in wild-type and Mkx ${ }^{-1-}$ TPCs, TBs, and TCs. ns no significance; ${ }^{*} P<0.05 ;{ }^{* * P}<0.01$; ${ }_{* * * * P}<0.0001$ 1. TPCs tendon progenitor cells, TBs tenoblasts, TCs tenocytes 
a

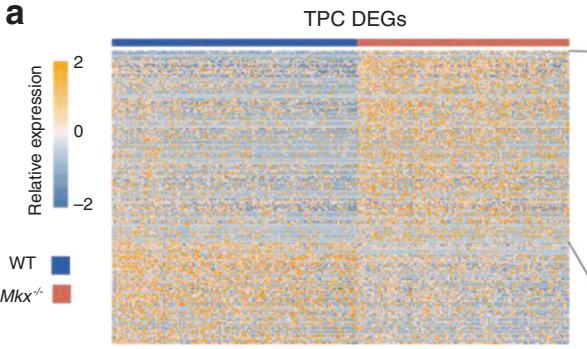

d

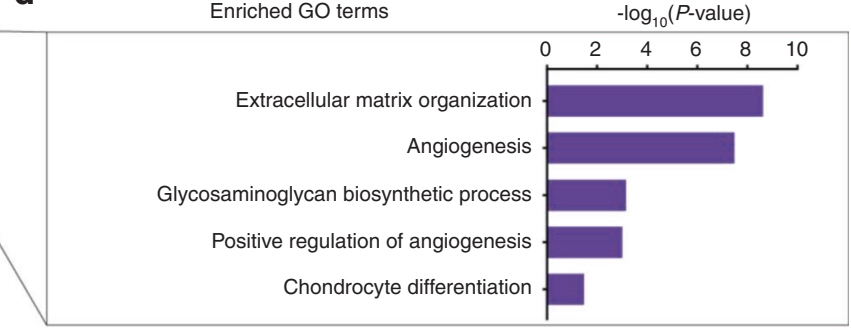

e

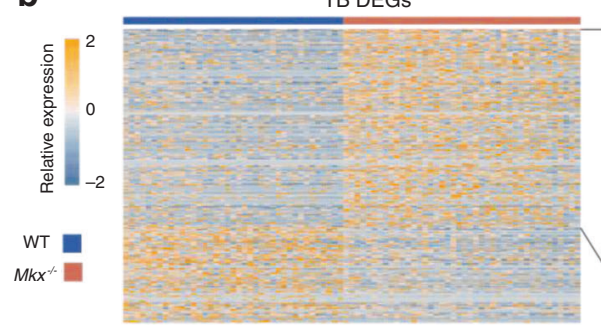
Enriched GO terms

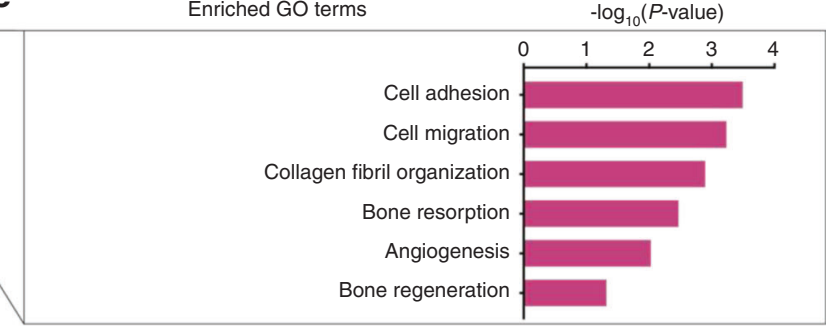

C

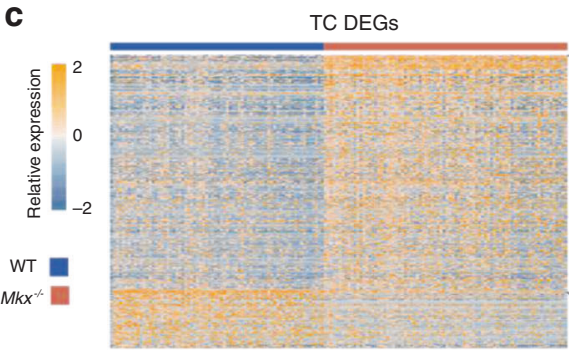

$\mathbf{f}$

Enriched GO terms $-\log _{10}(P$-value $)$

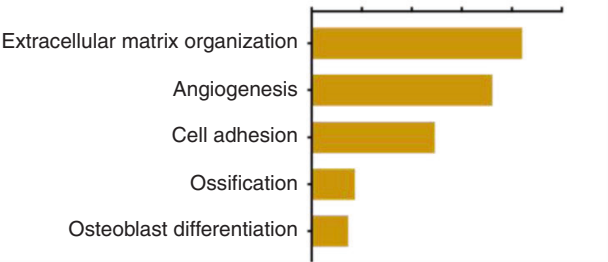

g
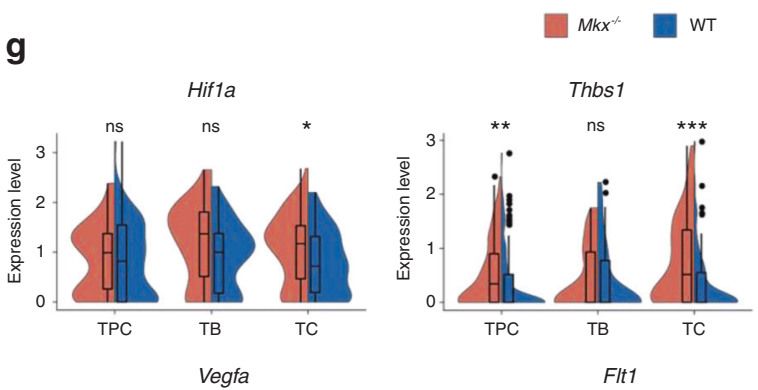

h

WT
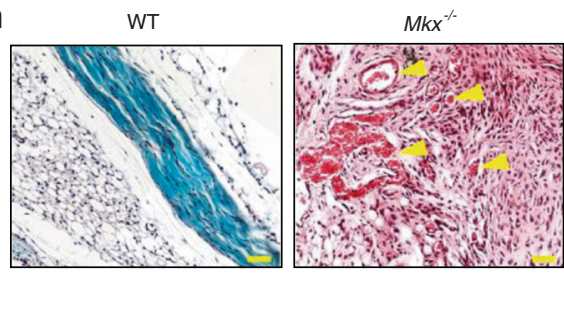

i
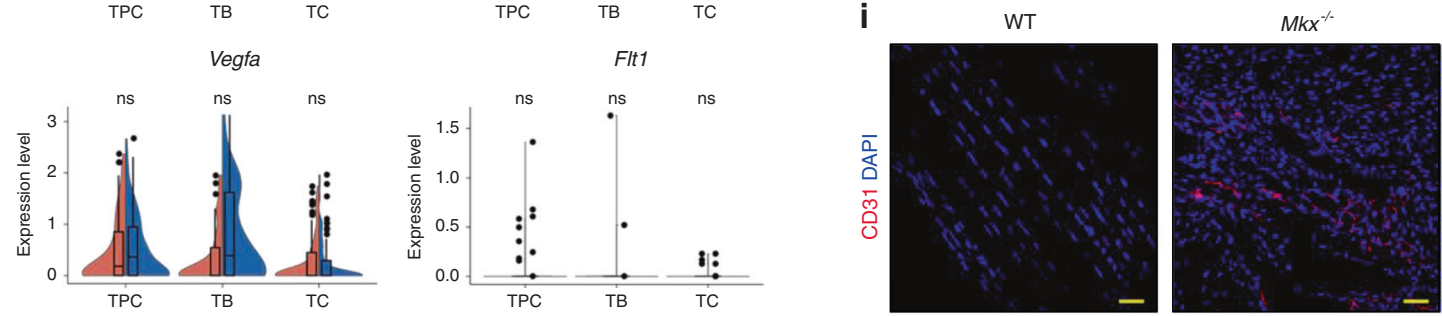

Fig. 5 Differential gene expression analysis revealed elevated angiogenic gene expression in $\mathrm{Mkx}^{-/-}$tendon cells. a-c Heatmap of the normalized gene expression of DEGs between WT and $M k x^{-1-}$ TPC (a), TB (b) and TC (c) clusters. d-f Representative GO terms corresponding to upregulated genes in the $M k^{-1-}$ TPC (d), TB (e) and TC (f) clusters. g Violin plots of proangiogenic genes, including Hif1a, Thbs 1, Vegfa, and Flt 1, in the TPC, TB and TC clusters. $\mathbf{h}$ Safranin O-Fast green staining of the patellar tendons from WT and $M \mathrm{kx}^{-1-}$ mice. The yellow arrows indicate the blood vessels. Scale bar, $50 \mu \mathrm{m}$. i Immunostaining of CD31 in WT and $M k^{-1-}$ patellar tendons. Scale bar, $50 \mu \mathrm{m}$. ns no significance; ${ }^{*} P<0.05 ;{ }^{*} P<0.01 ;{ }^{* *} P<0.001$. TPCs tendon progenitor cells, TBs tenoblasts, TCs tenocytes

three cell types of $M k^{-/-}$tendons (Fig. 5d-f), which was confirmed by our observations at the bulk level (Fig. 2b, d). Specifically, violin plots showed that Hifla was highly expressed in $M k x^{-1-}$ TCs, and Thbs 1 was highly expressed in $M k x^{-1-}$ TPCs and TCs (Fig. $5 \mathrm{~g}$ ). The expression of Vegfa and Flt 1 was comparable among each cell cluster, indicating that Vegfa/Vegfr signaling was not activated at this stage (Fig. $5 \mathrm{~g}$ ). SOFG and immunofluorescence staining of CD31 also showed increased blood vessels in $\mathrm{Mkx}^{-1-}$ tendons (Fig. 5h, i). Altogether, these findings indicate that the progression of tendon $\mathrm{HO}$ is highly coupled with the upregulation of angiogenesis-related genes and increased vascularity. 
a

$M k x^{-/}$degenerative model of $\mathrm{HO}$

\begin{tabular}{|c|c|c|c|c|}
\hline Postnatal & Week 2 & Week 5 & Week 8 & Week 12 \\
\hline day & $\frac{1}{\mathrm{BIBF} 112}$ & $\stackrel{1}{\text { eatment }}$ & $\begin{array}{c}\mathrm{L} \\
\text { Collect } \\
\text { sample }\end{array}$ & $\begin{array}{c}\text { Collect } \\
\text { sample }\end{array}$ \\
\hline
\end{tabular}

b
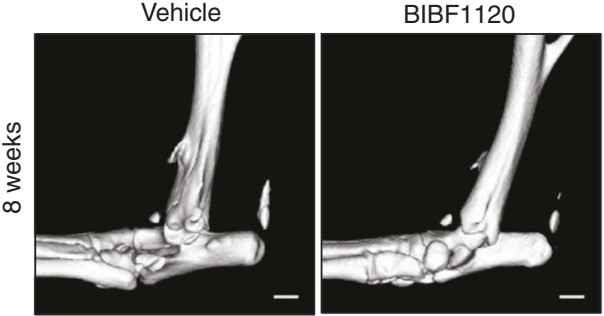

d
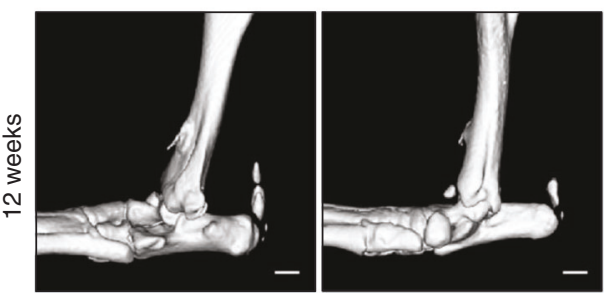

C

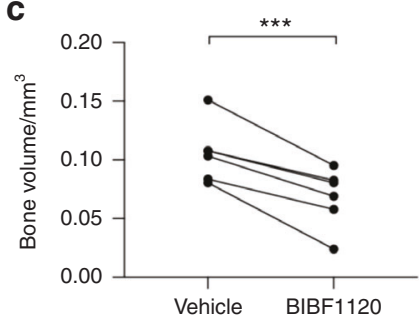

e

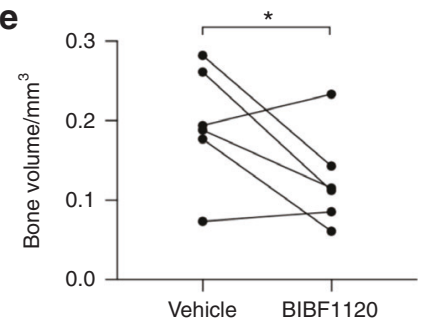

$\mathbf{f}$
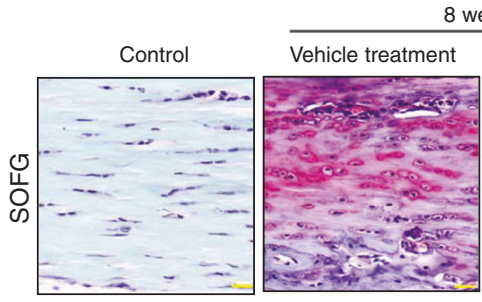

8 weeks
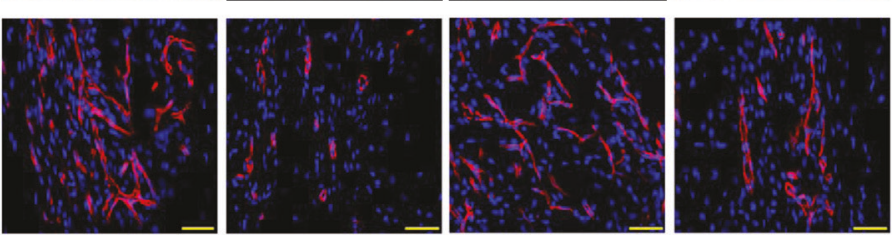

h
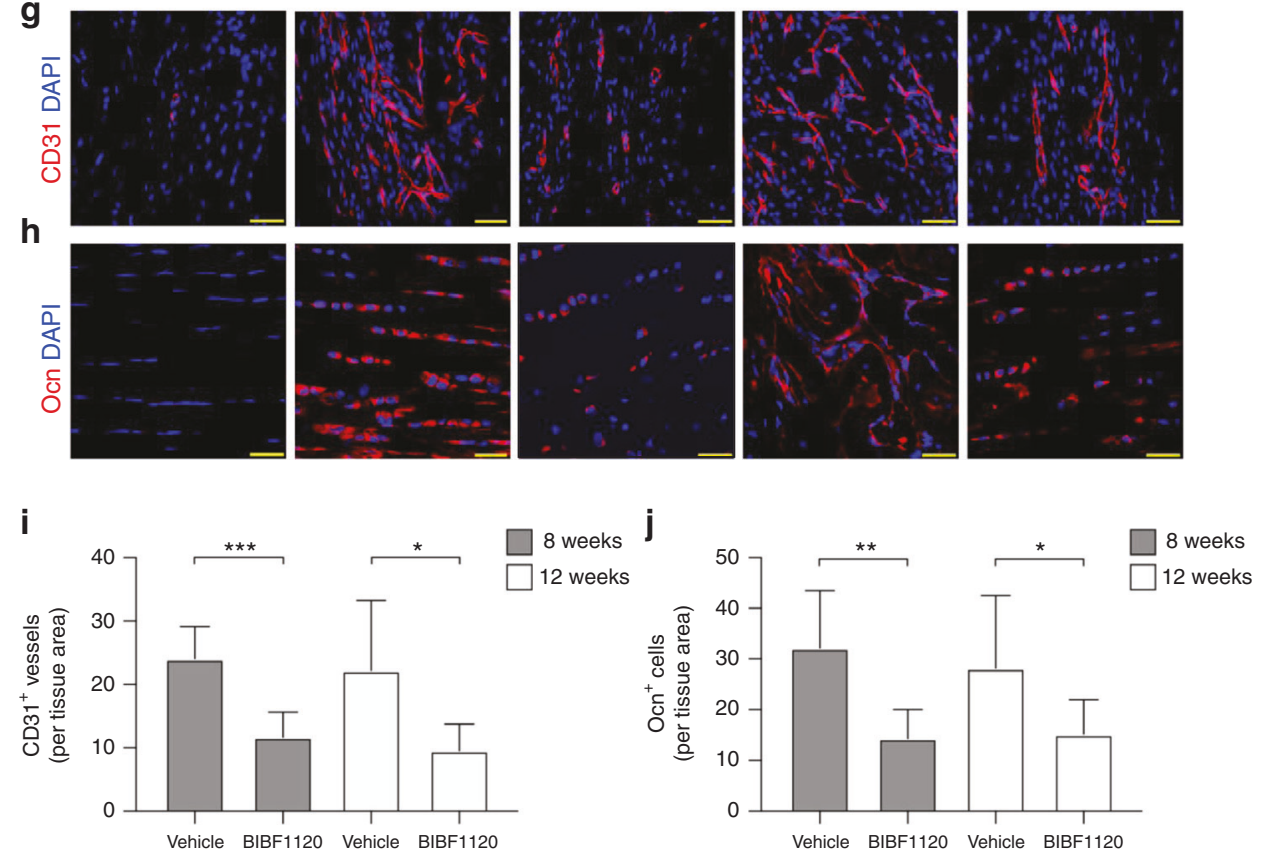

Fig. 6 The angiogenesis inhibitor BIBF1120 attenuates heterotopic bone formation in the $\mathrm{Mkx}^{-/-}$degenerative model of HO. a Schematic illustration of the treatment and sample collection workflow. b-e Micro-CT images of the Achilles tendons of 8-week-old (b) and 12-week-old (d) $\mathrm{Mkx}^{-1-}$ mice treated with BIBF1120 or vehicle. Scale bar, $1 \mathrm{~mm}$. Quantitative analysis of the heterotopic bone volumes of the Achilles tendons of 8-week-old (c) and 12-week-old (e) $\mathrm{Mkx}^{-1-}$ mice. f Safranin O-Fast green staining of the Achilles tendons of normal, 8-week-old and 12 -week-old $\mathrm{Mkx}^{-1-}$ mice. Scale bar, $50 \mu \mathrm{m}$. g Immunostaining of $\mathrm{CD}^{+} 1^{+}$(red) vessels. Scale bar, $50 \mu \mathrm{m}$. $\mathbf{h}$ Immunostaining of Ocn ${ }^{+}$(red) cells in ectopic bone marrow. Scale bar, $25 \mu \mathrm{m}$. i, j Quantification of CD31 ${ }^{+}$(i) vessels and $\mathrm{Ocn}^{+}$(j) cells. All data are shown as the mean \pm s.d. $n=6$ per group. Statistical test: student $t$ test. ${ }^{*} P<0.05 ;{ }^{* *} P<0.01 ;{ }^{* * *} P<0.001$ 
Rat injury-induced model of $\mathrm{HO}$

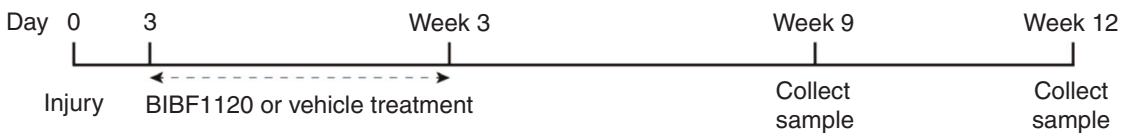

b

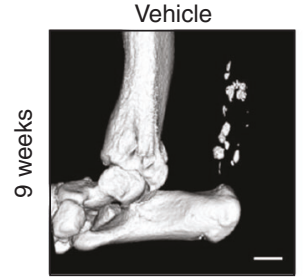

d
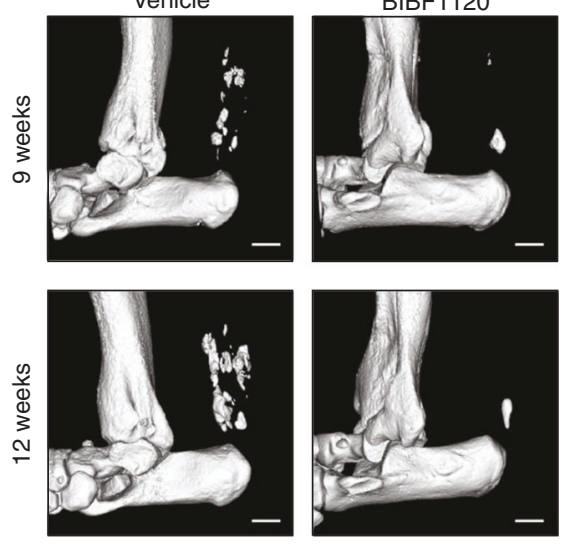

f

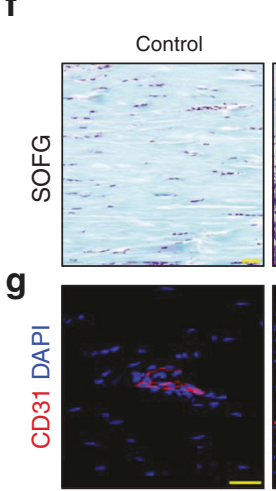

h
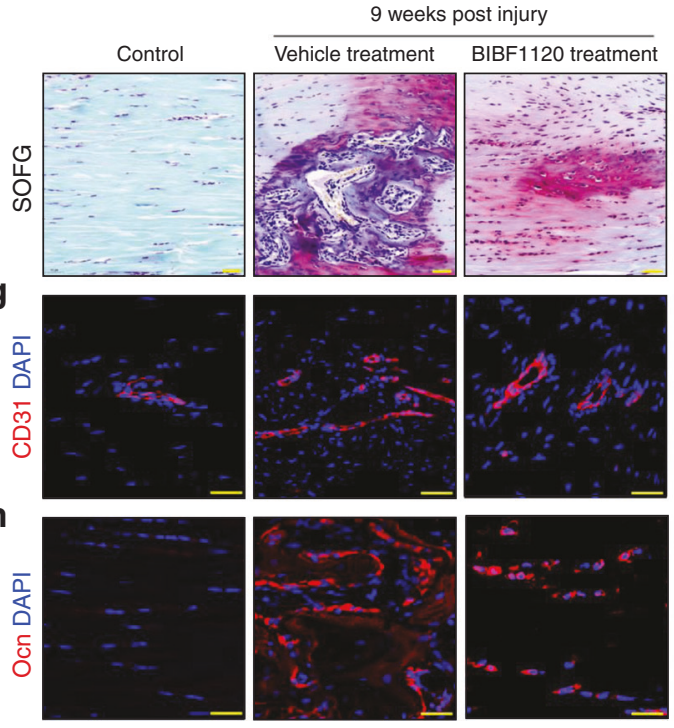

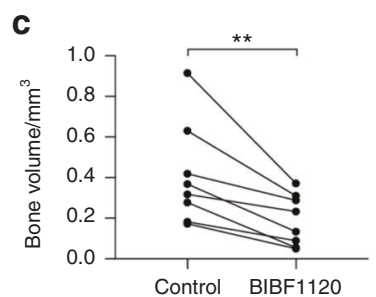

e

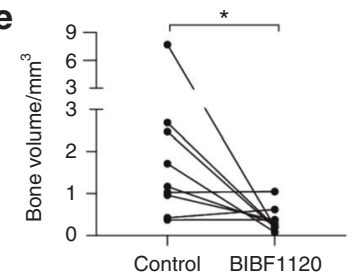

12 weeks post injury
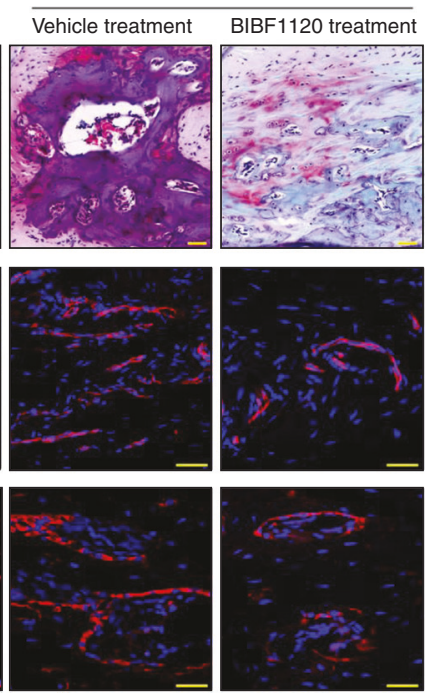

i
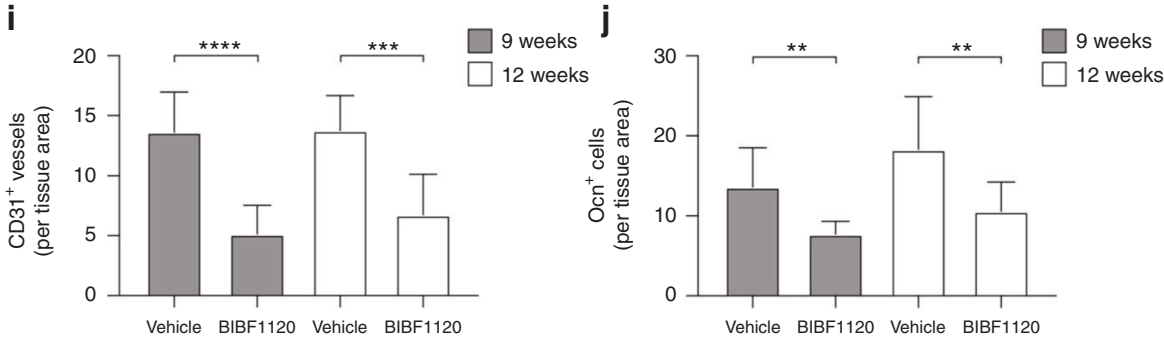

Fig. 7 The angiogenesis inhibitor BIBF1120 attenuates heterotopic bone formation in a trauma-induced model of rat HO. a Schematic illustration of the treatment and sample collection workflow for the rat HO model. $\mathbf{b}$-e Micro-CT images of the Achilles tendons of rats in the BIBF1120 and vehicle treatment groups at 9 (b) and 12 weeks (d) post ATP. Scale bar, $1 \mathrm{~mm}$. Quantitative analysis of the heterotopic bone volumes of the Achilles tendons at 9 (c) and 12 weeks (e) postinjury. $\mathbf{f}$ Safranin O-Fast green staining of the rat Achilles tendons of normal rats at 9 and 12 weeks postinjury. Scale bar, $50 \mu \mathrm{m}$. g Immunostaining of CD31 ${ }^{+}$(red) vessels. Scale bar, $50 \mu \mathrm{m}$. h Immunostaining of Ocn ${ }^{+}$(red) cells in ectopic bone marrow. Scale bar, $25 \mu \mathrm{m}$. i, j Quantification of CD31 ${ }^{+}$(i) vessels and $\mathrm{Ocn}^{+}$(j) cells. All data are shown as the mean \pm s.d. $n=8$ per group. Statistic test: student $t$ test. ${ }^{* *} P<0.01 ;{ }^{* *} P<0.001 ;{ }^{* * *} P<0.0001$

The angiogenesis inhibitor BIBF1120 attenuates the progression of $\mathrm{MkX}^{-1-}$ and trauma-induced $\mathrm{HO}$

We next investigated whether the inhibition of angiogenesis would attenuate $\mathrm{HO}$ progression. The angiogenesis inhibitor
BIBF1120 or vehicle (solvent used to dissolve BIBF1120, as a negative control) was injected into the Achilles tendons of $M k x$ knockout mice three times a week for three consecutive weeks from postnatal day 14 . The mice were sacrificed at the age of 8 or 
12 weeks for further characterization (Fig. 6a). The micro-CT results showed that bone formation was significantly mitigated by BIBF1120 treatment at both time points (Fig. 6b-e). SOFG staining revealed larger and well-developed cancellous bones and marrow in the vehicle group (Fig. 6f). Significantly more $\mathrm{CD} 31^{+}$ vessels were observed in the vehicle group than in the BIBF1120 group (Fig. $6 \mathrm{~g}, \mathrm{i}$ ). In addition, the $\mathrm{Ocn}^{+}$osteoblast number was significantly lower in the BIBF1120 group than in the vehicle group (Fig. $6 \mathrm{~h}, \mathrm{j}$ ). Altogether, these results indicated that the inhibition of angiogenesis by BIBF1120 alleviated HO progression in $M k x^{-/-}$tendons.

However, the $\mathrm{Mkx}^{-1-} \mathrm{HO}$ model represents a degenerative model in which the animals were not subjected to any trauma. However, in the clinic, tendon $\mathrm{HO}$ is often caused by trauma ${ }^{25}$. To better simulate clinical tendon $\mathrm{HO}$, we constructed a traumainduced $\mathrm{HO}$ model in rats based on percutaneous Achilles tendon puncture (ATP) ${ }^{3}$. We found that the expression of Mkx was also downregulated in the trauma-induced $\mathrm{HO}$ model (Supplementary Fig. S5). We then treated the trauma-induced $\mathrm{HO}$ model rats with BIBF1120 or vehicle. Each rat received a subcutaneous injection (between the Achilles tendon and skin) of BIBF1120 or vehicle three times a week for three weeks from the third day after injury and was sacrificed at 9 or 12 weeks postinjury (Fig. 7a). Micro-CT and SOFG staining analyses showed that the HO formation and bone volume were significantly reduced in the BIBF1120-treated group compared with the control group at 9 and 12 weeks after ATP (Fig. 7b-f). Importantly, the immunofluorescence staining results showed that $\mathrm{CD} 31^{+}$vessels were reduced in the BIBF1120 group relative to the vehicle control group (Fig. $7 \mathrm{~g}$, i). In addition, the number of $\mathrm{Ocn}^{+}$osteoblasts was significantly reduced upon BIBF1120 treatment (Fig. 7h, j). Collectively, these results demonstrated that the inhibition of angiogenesis with the small molecule BIBF1120 attenuated $\mathrm{HO}$ progression.

However, BIBF1120 targets various signaling pathways, including the Vegfr, Pdgfr and Fgfr signaling pathways, which are also important for the maintenance of tendon homeostasis ${ }^{26-28}$. To determine whether BIBF1120 treatment affects tendon cell proliferation and differentiation, we performed a bromodeoxyuridine (BrdU) assay and RNA-seq analysis of rat tendons treated with BIBF1120 and vehicle. Immunofluorescence staining showed similar levels of BrdU-positive cells in the BIBF1120- and vehicletreated groups (Supplementary Fig. S6a, b). Consistently, the examination of proliferation marker genes (Mki67, Pcna, Mcm2) showed that their expression levels were comparable between the BIBF1120 and vehicle groups (Supplementary Fig. S6d). In addition, the gene expression levels at the genome-wide scale were highly correlated between the BIBF1120- and vehicle-treated groups, suggesting that BIBF1120 minimally effects the transcript profiles of tendons (Supplementary Fig. S6c). Importantly, we also observed similar expression levels of tendon stem/progenitor cell markers (Mcam, Thy1, Nes) and other canonical tendon markers (Scx, Mkx, Egr1, Tnmd, Tnc and Col1a1) between the BIBF1120 and vehicle groups, indicating that tendon homeostasis and differentiation were not obviously affected by BIBF1120 (Supplementary Fig. S6e).

\section{DISCUSSION}

The limited understanding of tendon $\mathrm{HO}$ and the urgent need for an effective therapeutic motivated us to investigate its pathogenesis and to develop potential novel therapeutic strategies. Here, we used $M k^{-1-}$ mice as the tendon $\mathrm{HO}$ model to study the underlying mechanisms. Our data revealed a previously unknown role of $M k x$ in regulating the fates of different cell subpopulations. These high-throughput sequencing data suggested the inhibition of angiogenesis as a potential therapeutic target for attenuating tendon $\mathrm{HO}$, which inspired us to assess the effect of the small-molecule angiogenesis inhibitor BIBF1120 on the progression of tendon HO. Our data demonstrated that treatment with BIBF1120 displayed promising effects on attenuating the progression of degenerative and trauma-induced tendon HO.

Recently, an increasing number of studies have used singlecell analysis to explore the roles of different cell subpopulations in tendon development and pathology ${ }^{7,8,29}$. Using the highly parallel strategy of single-cell quantitative reverse transcription polymerase chain reaction (single-cell qRT-PCR), we previously identified three subpopulations of human tendon stem/progenitor cells (TSPCs) after in vitro expansion and confirmed that the Nestin-positive subpopulation possesses the best tendon regeneration capability ${ }^{7}$. Our single-cell analyses presented herein did not recapitulate the cell types we previously identified due to the species differences and to the potential selective effects of in vitro culture on the cell subpopulations. Although our data indicated that TPCs may gain osteogenic differentiation potential upon $M k x$ knockout, further studies are needed to trace their precise fate. Moreover, other cell populations, such as endothelial cells, smooth muscle cells and pericytes, which are important for angiogenesis, were not identified in our single-cell analysis, probably due to the limited number of cells captured. Further analyses using a high-throughput single-cell method may provide a more comprehensive landscape of the transcriptional heterogeneity of tendon cells under normal and heterotopic ossification conditions.

Angiogenesis and osteogenesis processes are highly coupled and are both critical for skeletal development ${ }^{30}$. However, neovascularization may have adverse effects on tissue repair. A recent study showed that the excessive activation of TGF- $\beta$ signaling promoted osteogenesis and neovascularization during $\mathrm{HO}$ progression ${ }^{3}$. Cocks and colleagues analyzed 29 human $\mathrm{HO}$ specimens and found that human $\mathrm{HO}$ exhibited a time- and space-dependent pattern of vascularization ${ }^{31}$. Our results showed that angiogenesis-related genes were upregulated in an $\mathrm{MkX}^{-1-} \mathrm{HO}$ mouse model, and neovascularization was validated by histological analyses. Thus, we hypothesized that inhibiting neovascularization may attenuate tendon heterotopic ossification. In this study, we found that the smallmolecule drug BIBF1120 significantly inhibited bone formation and vascularity in both $M k^{-1-}$ mice and a trauma-induced model of HO. BIBF1120 is a triple vascular kinase inhibitor that can effectively suppress the proangiogenic signaling pathway mediated by Vegfr, Pdgfr and Fgfr in vascular endothelial cells, perivascular skin cells and smooth muscle cells ${ }^{26}$. In addition to mediating the proangiogenic signaling pathway, vegfr, pdgfr, and fgfr are involved in signaling pathways that regulate many aspects of cell biology, such as cell proliferation, migration and fate determination ${ }^{32-34}$. In tendon biology, for example, Fgf/ Fgfr was the first signaling pathway identified to induce tendon cells in vivo, and Pdgf/Pdgfr signaling plays an important role in postnatal tendon growth ${ }^{27,28}$. Thus, the paninhibition of these signaling pathways by BIBF1120 could have adverse effects on surrounding tendon cells that are not specific to vascular blockade. Nevertheless, our results showed that the treatment of tendons with BIBF1120 had minimal side effects on the proliferation and expression of key marker genes, showing a relatively specific function of BIBF1120 in preventing tendon $\mathrm{HO}$ and highlighting its promising application potential.

In summary, this study revealed the mechanism underlying tendon $\mathrm{HO}$ by using an Mkx knockout model and potential provides a clinically applicable therapeutic approach to attenuate tendon $\mathrm{HO}$ through the inhibition of angiogenesis with BIBF1120. These findings will be of great value for elucidating the pathogenesis of tendon $\mathrm{HO}$ as well as for the development of novel therapeutic approaches for heterotopic ossification induced by tendon injury and surgery. 


\section{MATERIALS AND METHODS}

Animals

$M \mathrm{kx}^{-1-}$ transgenic mice were provided by Dr. Ronen Schweitzer (Oregon Health and Science University, Portland, OR). All animal procedures in this study were approved by the Institutional Animal Care and Use Committee of Zhejiang University.

\section{Histological and histochemical examination}

For histological analysis, samples were fixed in $4 \%(\mathrm{v} / \mathrm{v})$ paraformaldehyde for 24 hours and then decalcified in $10 \%(\mathrm{w} / \mathrm{v})$ EDTA-decalcification solution for 20 days. Achilles tendons were fixed in $4 \%(\mathrm{v} / \mathrm{v})$ paraformaldehyde for 24 hours. Then, the samples were dehydrated through an alcohol gradient, cleared, and embedded in paraffin blocks. Histological sections $(6 \mu \mathrm{m})$ were prepared using a microtome and subsequently processed for safranin O-fast green staining and immunostaining. Immunohistochemical analysis was performed in accordance with the standard protocol. Sections were incubated overnight at $4{ }^{\circ} \mathrm{C}$ with primary antibodies and then incubated at room temperature for $2 \mathrm{~h}$ with secondary antibodies. For immunofluorescence, the following antibodies were used: anti-MKX (1:100, LifeSpan, LS-B8063), antiOCN (1:100), anti-FSTL1 (1:100, Proteintech, 20182-1-AP), antiITM2A (1:100, Proteintech, 18306-1-AP), anti-COL1 (1:100, Abcam, ab34710), anti-CD31 (1:100, Abcam, ab222783), anti-BrdU (1:150, Proteintech, 66241-1-lg), and secondary antibodies conjugated with Alexa Fluor 488 and 546 (1:500, Thermo Fisher Scientific). The stained specimens were photographed digitally under a fluorescence microscope.

Microcomputed tomography imaging and analyses

Hind limbs and knee joints were dissected from mice and rats, fixed overnight in $4 \%$ paraformaldehyde and analyzed by MILabs U-SPECT4CT with default parameters.

Bulk RNA-seq and data analysis

For the bulk population, total RNA was extracted from tail tendons using TRIzol reagent (Invitrogen, Carlsbad, CA, USA), and a CDNA library was prepared using the lon Total RNA-Seq Kit v2 (Life Technologies Corporation, Carlsbad, USA) according to the manufacturer's protocol. RNA sequencing was performed on the Ion Proton system. Clean reads were obtained from the raw reads by removing the adaptor sequences, reads with $>5 \%$ ambiguous bases (noted as $\mathrm{N}$ ) and low-quality reads containing more than $20 \%$ of bases with a quality score $<13$. The clean reads were then aligned to the mouse genome (mm9) using the MapSplice program (v2.1.6). We applied the DESeq2 algorithm to filter the differentially expressed genes with the following criteria: (i) $\log 2$ (fold change) $>1$ or $<-1$ and (ii) padj $<0.05$. Gene ontology (GO) analysis was performed using DAVID ${ }^{35,36}$.

Single-cell capture, cDNA library preparation and sequencing First, tail tendons from 4-week-old WT or $\mathrm{Mkx}^{-1-}$ mice were minced into desired pieces and digested with $0.2 \%$ collagenase I (Life Technologies) diluted in low-glucose Dulbecco's modified Eagle's medium (Gibco) at $37^{\circ} \mathrm{C}$ for $2 \mathrm{~h}$. The resulting cell suspension was filtered using a $40 \mu \mathrm{m}$ strainer to remove the incompletely digested clumps and adjusted to a concentration of $4 \times 10^{5}$ cells per $\mathrm{mL}$. Single-cell capture, RNA extraction and cDNA preparation were performed in accordance with methods described in the Fluidigm protocol (PN 100-9886, Using C1 High-Throughput IFC to Generate Single-cell cDNA Libraries for mRNA Sequencing). The cDNA reaction products were quantified using a Qubit fluorometer and were then diluted to a final concentration of $0.2 \mathrm{ng} \cdot \mathrm{\mu L}^{-1}$ using $\mathrm{C} 1$ Harvest Reagent. The diluted CDNA reaction products were then converted into mRNAseq libraries using the Nextera XT DNA Sample Preparation Kit (Illumina, FC-131-1096, -2001 and -2002) in accordance with the manufacturer's instructions. The library was sequenced by
Annoroad (Beijing, China) on an Illumina HiSeq $X$ Ten platform (Illumina, Santiago, CA, USA), and $150 \mathrm{bp}$ paired-end reads were generated.

Single cell RNA-seq data analysis

Single-cell RNA sequencing reads were evaluated by $A f t e r Q C^{37}$. Bad reads were detected automatically and removed. Bases at the $3^{\prime}$ end of the read were further trimmed by NGSQCToolkit if their PHRED quality score was less than $20^{38}$. The reads were mapped to the $\mathrm{mm} 9$ genome using bowtie2. The mapped reads for genes were counted with featureCounts ${ }^{39}$. Quality control, clustering and differential expression analysis were performed using Seurat v2.3.0 in accordance with a recently published method ${ }^{16,40}$. For integrated analysis, the union set of the top 1000 highly variable genes in WT and $M k^{-/-}$cells was used to perform a canonical correlation analysis, and 20 cells that were not well described by a shared gene correlation structure were excluded. Four principal components were used for the alignment of the CCA subspaces and for cell clustering at a resolution of 0.4 . The marker genes of each cell cluster were analyzed using the FindConservedMarkers function with the test.use = "wilcox" option and the logfc.threshold parameter set to 0.5 . Cell type-specific differentially expressed genes were identified using the FindMarkers function with default parameters. Pseudotime analysis was performed with Monocle v2.6.3 using digital gene expression matrices with annotations from Seurat as the input ${ }^{24}$.

Gene set enrichment analysis

The module scores for gene expression programs in single cells were calculated using the AddModuleScore function of the Seurat package ${ }^{41}$. First, all the analyzed genes were binned based on the average expression, and the control genes were randomly selected from each bin. Then, the average expression value of the gene set at the single-cell level minus the aggregated expression of the control gene set was calculated. Gene sets were obtained from the Mgi database (http://www.informatics.jax.org/ mgihome/GO).

\section{Animal experiments}

For the $\mathrm{MkX}^{-1-}$ mouse $\mathrm{HO}$ model, each 2-week-old mouse was subcutaneously injected (between the Achilles tendon and skin) with $8 \mu \mathrm{L}$ of BIBF1120 $(4 \mu \mathrm{g})$ in the right leg and $8 \mu \mathrm{L}$ of vehicle (an identical solvent used to dissolve BIBF1120) in the left leg three times a week for three weeks. The mice were euthanized at 8 or 12 weeks old for micro-CT imaging and histological analysis.

For the rat traumatic model of $\mathrm{HO}$, eight-week-old male rats were anesthetized by isoflurane. A 27-gauge needle was used to puncture the Achilles tendon body from the lateral aspect percutaneously, and this process was repeated twenty-five times at five different parts of the Achilles tendon body for each rat. Three days postinjury, each rat was subcutaneously injected with $40 \mu \mathrm{L}$ of BIBF1120 $(40 \mu \mathrm{g})$ in the right leg and $40 \mu \mathrm{L}$ of vehicle in the left leg three times a week for three weeks. At 9 or 12 weeks after treatment, the rats were euthanized for micro-CT imaging and histological analysis.

Cell proliferation in vivo was detected as previously described $^{42}$. Specifically, BrdU was reconstituted in DMSO as a stock $\left(80 \mathrm{mmol} \cdot \mathrm{L}^{-1}\right)$, and $150 \mu \mathrm{L}$ of BrdU mixed with $350 \mu \mathrm{L}$ of PBS was injected intraperitoneally into the rats together with BIBF1120 and the vehicle. Tissue sections were treated with $1.5 \mathrm{~mol} \cdot \mathrm{L}^{-1} \mathrm{HCl}$ at room temperature for $0.5 \mathrm{~h}$, and DNA synthesis was assessed with an anti-BrdU antibody.

Statistical analysis

All statistical analyses were performed with GraphPad Prism software. The means and standard deviations were calculated from numerical data and are presented in the text and figures. Significant differences in the mean values were determined using 
Student's $t$ test unless indicated otherwise. $P<0.05$ was considered significant. Statistical significance is indicated by ${ }^{*} P<0.05$, ${ }^{* *} P<0.01$, ${ }^{* * *} P<0.001$, and ${ }^{* * *} P<0.0001$.

\section{DATA AVAILABILITY}

The single-cell and bulk RNA-seq data utilized in this study are deposited into the Gene Expression Omnibus (GEO) under accession number GSE102931.

\section{ACKNOWLEDGEMENTS}

We thank Dr. Savio L-Y. Woo and Dr. Mikael Bjorklund for the helpful advice regarding the writing of the manuscript. This work was supported by the National Key R\&D Program of China (2017YFA0104900), the National Natural Science Foundation of China (31830029, 81501937 and 81522029), and the Fundamental Research Funds for the Central Universities (K20200099).

\section{AUTHOR CONTRIBUTIONS}

Conceptualization, J.L., X.C., and H.W.O.; investigation, J.L., Y.Y., W.Z., C.D., Y.X., S.H., H.L., Y.H., and C.T.; formal analysis, J.L., W.L., and D.Z.; visualization, J.L., W.Z., W.L., and Y.C.; writing - original draft, J.L., W.Z. and W.L.; writing-review and editing, W.L., D.C., B.V., and H.W.O.; funding acquisition, W.L., X.Z., and H.W.O.; supervision, W.L and H.W.O.

\section{ADDITIONAL INFORMATION}

Supplementary information The online version contains supplementary material available at https://doi.org/10.1038/s41413-021-00175-9.

Competing interests: The authors declare no competing interests.

\section{REFERENCES}

1. Shehab, D., Elgazzar, A. H. \& Collier, B. D. Heterotopic ossification. J. Nucl. Med 43, 346-353 (2002).

2. Meyers, C. et al. Heterotopic ossification: a comprehensive review. JBMR Plus $\mathbf{3}$, e10172 (2019)

3. Wang, X. et al. Inhibition of overactive TGF-beta attenuates progression of heterotopic ossification in mice. Nat. Commun. 9, 551 (2018).

4. Agarwal, S. et al. Scleraxis-lineage cells contribute to ectopic bone formation in muscle and tendon. Stem Cells 35, 705-710 (2017).

5. $\mathrm{Bi}, \mathrm{Y}$. et al. Identification of tendon stem/progenitor cells and the role of the extracellular matrix in their niche. Nat. Med. 13, 1219-1227 (2007).

6. Lee, C. H. et al. Harnessing endogenous stem/progenitor cells for tendon regeneration. J. Clin. Invest 125, 2690-2701 (2015).

7. Yin, Z. et al. Single-cell analysis reveals a nestin+tendon stem/progenitor cell population with strong tenogenic potentiality. Sci. Adv. 2, e1600874 (2016).

8. Harvey, T., Flamenco, S. \& Fan, C. M. A Tapp $3^{+}$Pdgfra $^{+}$tendon stem cell population contributes to regeneration and reveals a shared role for PDGF signalling in regeneration and fibrosis. Nat. Cell Biol. 21, 1490-1503 (2019).

9. Ito, Y. et al. The Mohawk homeobox gene is a critical regulator of tendon differentiation. Proc. Natl. Acad. Sci. USA 107, 10538-10542 (2010).

10. Kimura, W. et al. Irxl1 mutant mice show reduced tendon differentiation and no patterning defects in musculoskeletal system development. Genesis 49, 2-9 (2011).

11. Liu, H. et al. Mohawk promotes the tenogenesis of mesenchymal stem cells through activation of the TGFbeta signaling pathway. Stem Cells 33, 443-455 (2015).

12. Liu, W. et al. The atypical homeodomain transcription factor Mohawk controls tendon morphogenesis. Mol. Cell Biol. 30, 4797-4807 (2010).

13. Suzuki, $\mathrm{H}$. et al. Gene targeting of the transcription factor Mohawk in rats causes heterotopic ossification of Achilles tendon via failed tenogenesis. Proc. Natl. Acad. Sci. USA 113, 7840-7845 (2016).

14. Liu, H., Xu, J. \& Jiang, R. Mkx-deficient mice exhibit hedgehog signalingdependent ectopic ossification in the achilles tendons. J. Bone Min. Res. 34, 557-569 (2019).

15. Dejana, E. The role of wnt signaling in physiological and pathological angiogenesis. Circ. Res 107, 943-952 (2010)

16. Butler, A., Hoffman, P., Smibert, P., Papalexi, E. \& Satija, R. Integrating single-cell transcriptomic data across different conditions, technologies, and species. Nat. Biotechnol. 36, 411-420 (2018).
17. Noack, S. et al. Periostin secreted by mesenchymal stem cells supports tendon formation in an ectopic mouse model. Stem Cells Dev. 23, 1844-1857 (2014).

18. Van den Plas, D. \& Merregaert, J. Constitutive overexpression of the integral membrane protein Itm2A enhances myogenic differentiation of $\mathrm{C} 2 \mathrm{C} 12$ cells. Cell Biol. Int. 28, 199-207 (2004).

19. Van den Plas, D. \& Merregaert, J. In vitro studies on Itm2a reveal its involvement in early stages of the chondrogenic differentiation pathway. Biol. Cell 96, 463-470 (2004).

20. Boeuf, S. et al. Enhanced ITM2A expression inhibits chondrogenic differentiation of mesenchymal stem cells. Differentiation 78, 108-115 (2009).

21. Wenstrup, R. J. et al. Regulation of collagen fibril nucleation and initial fibril assembly involves coordinate interactions with collagens $\mathrm{V}$ and $\mathrm{XI}$ in developing tendon. J. Biol. Chem. 286, 20455-20465 (2011).

22. Docheva, D., Hunziker, E. B., Fassler, R. \& Brandau, O. Tenomodulin is necessary for tenocyte proliferation and tendon maturation. Mol. Cell Biol. 25, 699-705 (2005).

23. Gehwolf, R. et al. Pleiotropic roles of the matricellular protein Sparc in tendon maturation and ageing. Sci. Rep. 6, 32635 (2016).

24. Qiu, X. et al. Single-cell mRNA quantification and differential analysis with Census. Nat. Methods 14, 309-315 (2017).

25. Zhang, Q., Zhou, D., Wang, H. \& Tan, J. Heterotopic ossification of tendon and ligament. J. Cell Mol. Med 24, 5428-5437 (2020).

26. Hilberg, F. et al. BIBF 1120: triple angiokinase inhibitor with sustained receptor blockade and good antitumor efficacy. Cancer Res. 68, 4774-4782 (2008).

27. Huang, A. H., Lu, H. H. \& Schweitzer, R. Molecular regulation of tendon cell fate during development. J. Orthop. Res. 33, 800-812 (2015).

28. Sugg, K. B. et al. Postnatal tendon growth and remodeling require plateletderived growth factor receptor signaling. Am. J. Physiol. Cell Physiol. 314 C389-C403 (2018).

29. Tan, G. K. et al. Tgfbeta signaling is critical for maintenance of the tendon cell fate. Elife 9, e52695 (2020).

30. Kusumbe, A. P., Ramasamy, S. K. \& Adams, R. H. Coupling of angiogenesis and osteogenesis by a specific vessel subtype in bone. Nature 507, 323-328 (2014).

31. Cocks, M. et al. Vascular patterning in human heterotopic ossification. Hum. Pathol. 63, 165-170 (2017).

32. Olsson, A. K., Dimberg, A., Kreuger, J. \& Claesson-Welsh, L. VEGF receptor signalling - in control of vascular function. Nat. Rev. Mol. Cell Biol. 7, 359-371 (2006).

33. Andrae, J., Gallini, R. \& Betsholtz, C. Role of platelet-derived growth factors in physiology and medicine. Genes Dev. 22, 1276-1312 (2008).

34. Ornitz, D. M. \& Itoh, N. The fibroblast growth factor signaling pathway. Wiley Interdiscip. Rev. Dev. Biol. 4, 215-266 (2015).

35. Huang da, W., Sherman, B. T. \& Lempicki, R. A. Bioinformatics enrichment tools: paths toward the comprehensive functional analysis of large gene lists. Nucleic Acids Res. 37, 1-13 (2009).

36. Huang da, W., Sherman, B. T. \& Lempicki, R. A. Systematic and integrative analysis of large gene lists using DAVID bioinformatics resources. Nat. Protoc. 4, 44-57 (2009).

37. Chen, S. et al. AfterQC: automatic filtering, trimming, error removing and quality control for fastq data. BMC Bioinforma. 18, 80 (2017).

38. Patel, R. K. \& Jain, M. NGS QC Toolkit: a toolkit for quality control of next generation sequencing data. PLoS One 7, e30619 (2012).

39. Liao, Y., Smyth, G. K. \& Shi, W. featureCounts: an efficient general purpose program for assigning sequence reads to genomic features. Bioinformatics 30, 923-930 (2014).

40. Satija, R., Farrell, J. A., Gennert, D., Schier, A. F. \& Regev, A. Spatial reconstruction of single-cell gene expression data. Nat. Biotechnol. 33, 495-502 (2015).

41. Zou, Z. et al. A single-cell transcriptomic atlas of human skin aging. Dev. Cell 56, 383-397 e388 (2021).

42. Ho, T. C. et al. PEDF-derived peptide promotes tendon regeneration through its mitogenic effect on tendon stem/progenitor cells. Stem Cell Res. Ther. 10, 2 (2019).

Open Access This article is licensed under a Creative Commons Attribution 4.0 International License, which permits use, sharing, adaptation, distribution and reproduction in any medium or format, as long as you give appropriate credit to the original author(s) and the source, provide a link to the Creative Commons license, and indicate if changes were made. The images or other third party material in this article are included in the article's Creative Commons license, unless indicated otherwise in a credit line to the material. If material is not included in the article's Creative Commons license and your intended use is not permitted by statutory regulation or exceeds the permitted use, you will need to obtain permission directly from the copyright holder. To view a copy of this license, visit http://creativecommons. org/licenses/by/4.0/.

(c) The Author(s) 2022 